\title{
电极距离和取向对哪掺杂带帽碳纳米管分子结中 负微分电阻的影响
}

\author{
赵朋 ${ }^{(1)}$, 刘德胜 ${ }^{(2)}$, 梁伟 ${ }^{(1)}$ \\ (1) 济南大学物理科学与技术学院, 济南 250022 ; \\ (2) 山东大学物理学院, 晶体材料国家重点实验室, 济南 250100 \\ E-mail: ss_zhaop@ujn.edu.cn
}

2011-10-03 收稿, 2011-11-28 接受

国家自然科学基金(11104115, 11074146)、山东省自然科学基金(ZR2009AL004)、济南大学博士基金(XBS1004)和中国科学院半导体研究所 半导体材料科学重点实验室开放课题(KLSMS-0908)资助

摘要 利用非平衡格林函数和第一性密度泛函理论, 研究了电极距离和相对取向对喼掺杂 带帽碳纳米管分子结中负微分电阻行为的影响. 结果显示, 负微分电阻行为强烈依赖于电极 距离和相对取向. 当电极距离为 $0.35 \mathrm{~nm}$ 且体系的对称性最高时, 可以产生最好的负微分电 阻行为.

\section{关键词}

碳纳米管

负微分电阻

非平衡格林函数

密度泛函理论
近年来, 单个分子表征和操控技 术的进步使得发展单分子器件成为可 能 $^{[1]}$. 在不同的单分子结中, 人们发现 了诸多令人感兴趣的物理现象, 包括 负微分电阻 $(\mathrm{NDR})^{[2 ~ 10]}$ 、单电子特 性 $^{[11]}$ 、高度非线性伏安特性 $(I-V)^{[12]}$ 、 整流 ${ }^{[13]}$ 等, 其中最突出的就是 NDR, 即电流随着外加偏压的增加先增加而 后降低的效应. NDR 是一种非常有用 的性质, 可用于分子开关、逻辑和存储 单元. 在很多体系中都发现了 NDR 行 为, 如硝基和氨基取代中间苯环的苯

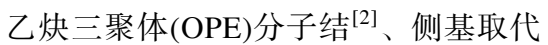
的卟啉分子结 ${ }^{[3]}$ 、葱分子结 ${ }^{[4]}$ 、金属和 半导体团簇 ${ }^{[5]}$ 、氮终端的单壁碳纳米管 $(\mathrm{SWCNT})$ 分子结 ${ }^{[6]} 、 \mathrm{C}_{121}{ }^{[7]}$ 和 $\mathrm{C}_{131}{ }^{[8]}$ 分 子结等.

在前面的工作中, 我们已经理论 研究了嗍掺杂带帽 SWCNT 分子结的 电子输运特性 ${ }^{[14]}$. 结果表明, 电子输 运性质强烈依赖于嗍掺杂的位置, 也
就是图 1 中的 $a, b, c, d$ 和 $e$ 位置. 当嗍 原子掺杂在针尖区域, 即 $a, b$ 和 $c$ 位置 时, 出现 NDR 行为. 特别地, $c$ 位置换 杂给出最高的峰谷比(PVR). 由于没有 中间桥接分子, 此 NDR 器件的结构相 较于普通 NDR 器件更加简单.

众所周知, 电极距离强烈影响分 子结的电子输运性质 ${ }^{[15,16]}$. 最近纳米 制造技术的进步使得人们可以精确控 制 SWCNT 电极间的空隙大小 ${ }^{[17]}$. 那 么, 就出现了一个基本但令人感兴趣 的问题: 电极距离是如何影响嗍掺杂 带帽 SWCNT 分子结中 NDR 行为的?
同时，电极间的相对取向对 NDR 行为 的影响也是令人感兴趣的 ${ }^{[15]}$. 本文利 用非平衡格林函数(NEGF)和第一性密 度泛函理论(DFT)相结合的方法, 进一 步研究这些效应.

图 1 为嗍掺杂带帽 $\operatorname{SWCNT}(5,5)$ 分子结示意图. 带帽 $\operatorname{SWCNT}(5,5)$ 的前 端通过半个富勒烯闭合. 嗍原子撸杂 在 $c$ 位置. 在理论模拟中, 分子结分为 三部分：左电极 $(\mathrm{L}) 、$ 中间散射区 $(\mathrm{C})$ 和 右电极 $(\mathrm{R})$. 两针尖之间的宽度 $w$ 分别 设为 $0.20,0.25,0.35$ 和 $0.40 \mathrm{~nm}$. 与我们 前面的工作一样 ${ }^{[14]}$, O 区利用 SIESTA

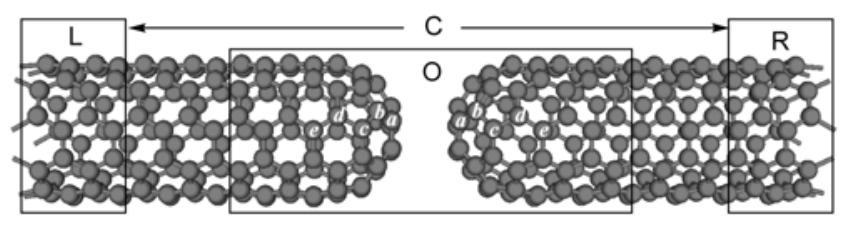

图 1 嗍掺杂带帽 SWCNT 分子结示意图 本研究中嗍原子占据 $c$ 位

英文版见: Zhao P, Liu D S, Liang W. Effects of tip separation and orientation on negative differential resistance in boron-doped carbon-nanotube-based molecular junctions. Chin Sci Bull, 2012, 57, doi: 10.1007/s11434-012-4972-y 
软件来进行结构优化 ${ }^{[18]}$. 输运计算采 用 ATK 软件 ${ }^{[19,20]}$, ATK 基于 NEGF 和 DFT.

图 2 给出了计算得到的不同针尖 距离下的 $I-V$ 曲线, 偏压范围为 $0 \sim 1.5 \mathrm{~V}$, 这一偏压范围在实际实验测量中是合 理的 ${ }^{[21]}$. 从 $I-V$ 曲线可以看出, 随着距 离的增加, 电流明显降低. 另外, 电极 距离显著影响 NDR 行为: 随着距离 $w$ 从 $0.35 \mathrm{~nm}$ 减小到 $0.25 \mathrm{~nm}, \mathrm{PVR}$ 变得
越来越弱, $w=0.20 \mathrm{~nm}$ 时, NDR 效应消 失; 当距离 $w$ 从 $0.35 \mathrm{~nm}$ 增大到 $0.40 \mathrm{~nm}$, PVR 稍微降低. 因此, 根据峰值高度 和 PVR 大小, $0.35 \mathrm{~nm}$ 的电极距离可以 产生相对好的 NDR 行为.

为了理解距离对 NDR 行为的影 响, 我们分别计算了 $w=0.20$ 和 0.35 $\mathrm{nm}$ 分子结在 $0,0.20,0.55,0.90$ 和 $1.20 \mathrm{~V}$ 时的透射谱, 如图 3 所示. 通过分子结 的电流由 Landauer-Büttiker ${ }^{[22]}$ 公式
给出:

$$
I=(2 e / h) \int_{\mu_{\mathrm{L}}}^{\mu_{\mathrm{R}}}\left(f_{\mathrm{L}}-f_{\mathrm{R}}\right) T(E, V) \mathrm{d} E,
$$

其中 $\mu_{\mathrm{L}, \mathrm{R}}=E_{\mathrm{F}} \mp \mathrm{eV} / 2, E_{\mathrm{F}}$ 是费米能级, $f$ 是费米分布函数, $T(E, V)$ 是与偏压有关 的透射函数. 因此, 只有能量在 $\left[\mu_{\mathrm{L}}, \mu_{\mathrm{R}}\right]$ 区间的电子才对总电流有贡献，这一 区域被称为偏压窗口或积分窗口。考 虑到 $E_{\mathrm{F}}$ 已经被设置为 $0 \mathrm{eV}$, 偏压窗口 实际上就是 $[-V / 2, V / 2]$. 于是, 电流就
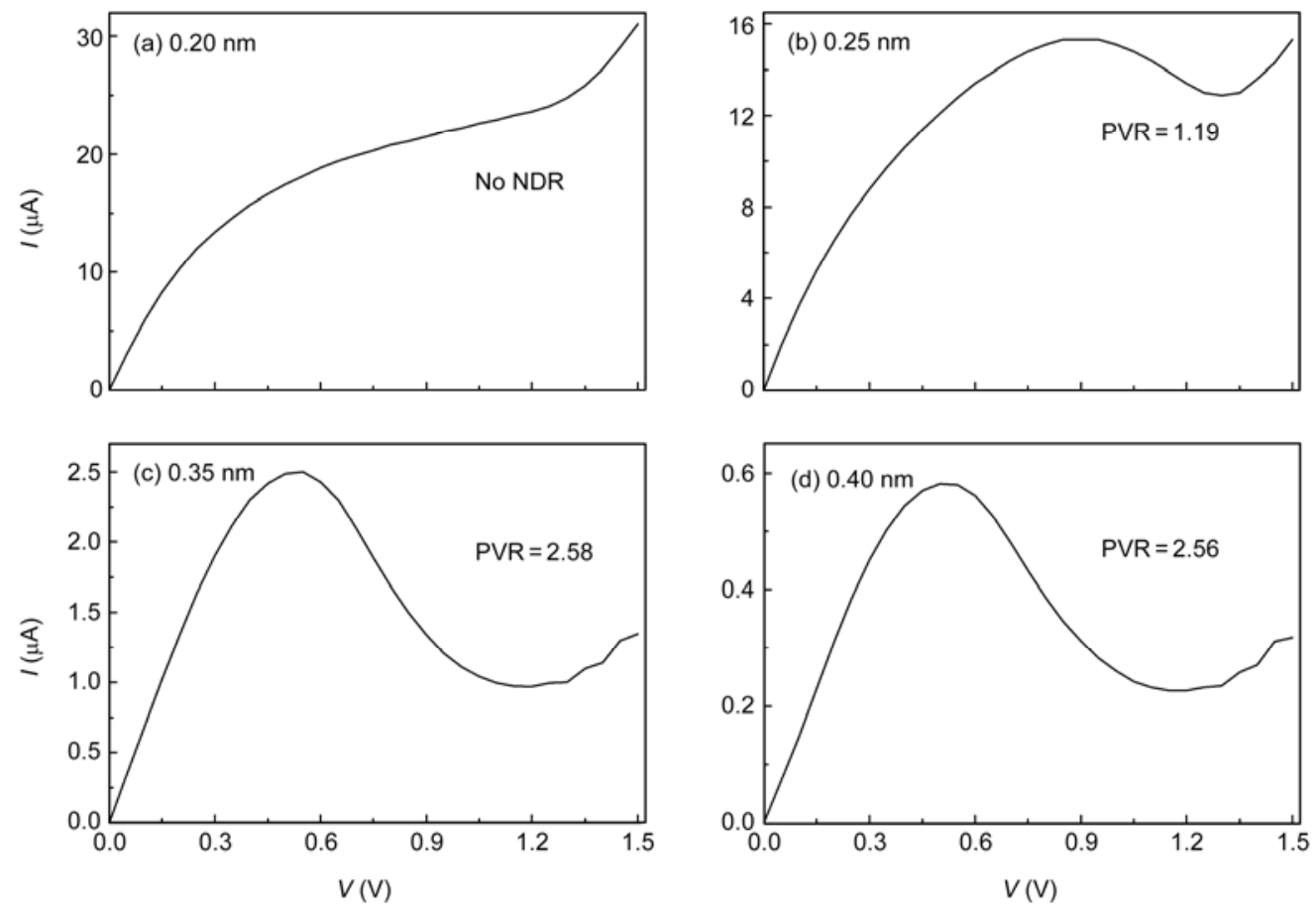

图 2 不同针尖宽度下嗍掺杂带帽 SWCNT 分子结的 $I-V$ 曲线

(a) 1
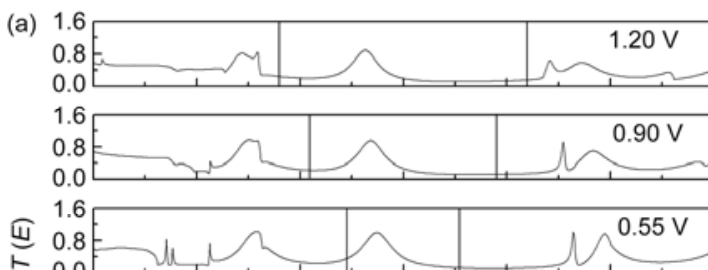

$\sim 0.0$

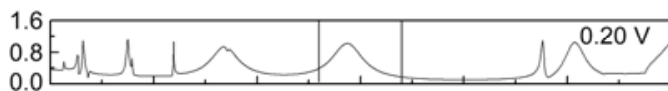

$$
0.0
$$

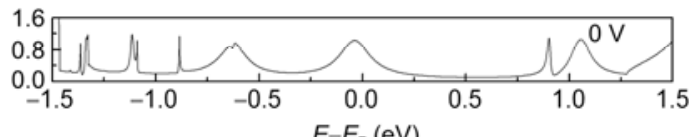

(b)

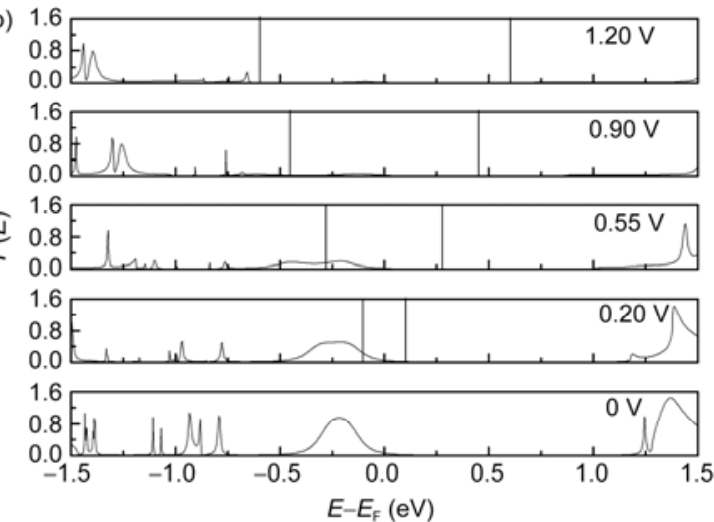

图 3 计算得到的不同偏压下不同电极距离分子结的透射谱

(a) $w=0.20 \mathrm{~nm}$; (b) $w=0.35 \mathrm{~nm}$. 两坚直实线之间的区域为偏压窗口 
是由偏压窗口内 $T(E, V)$ 的积分面积决 定的. 在 $w=0.20 \mathrm{~nm}$ 的分子结的偏压 窗口内总是有一个强而宽的透射峰(图) 3(a)), 且该峰几乎不随偏压的增加而 发生变化. 因此, 电流始终在增强, 没 有 NDR 行为. 小偏压下, $w=0.35 \mathrm{~nm}$ 的分子结的偏压窗口内 $E_{\mathrm{F}}$ 以下也有一 个强而宽的透射峰(图 3(b)), 导致电 流最初的增加. 然而外加偏压同时降 低了透射峰的高度. 当偏压超过 $0.55 \mathrm{~V}$ 时, 总透射峰高度的减小超过了不断 增大的偏压窗口, 导致电流的净减小, 从而引起了 NDR 现象.

为了理解不同电极距离分子结的 不同透射特性, 在 $0 \sim 1.20 \mathrm{~V}$ 偏压下, 我们分别将态密度投影到 $w=0.20$ 和 $0.35 \mathrm{~nm}$ 两分子结 $\mathrm{C}$ 区的左管(实线)和 右管(虚线) $)^{[7]}$, 如图 4 所示. 从投影态 密度谱(PDOS) 可以看到, 在没有施加 偏压时, 两套 PDOS 峰在能量上是简 并的. $E_{\mathrm{F}}$ 以下明显的 PDOS 峰产生了图 3 中 $E_{\mathrm{F}}$ 附近强而宽的透射峰. 当施加 $1.20 \mathrm{~V}$ 偏压时, $w=0.20 \mathrm{~nm}$ 分子结的两 套 PDOS 峰几乎仍然在能量上是简并 的(图 4(a)). 因此, 偏压窗口内强而宽 的透射峰几乎不随偏压增加而发生变 化(图 3(a)). 相反, 在 $1.20 \mathrm{~V}$ 偏压下, $w$ $=0.35 \mathrm{~nm}$ 分子结的两套 PDOS 峰在 $E_{\mathrm{F}}$ 附近不再简并(图 4(b)). 因此, 在 $E_{\mathrm{F}}$ 附 近就几乎没有任何明显的透射峰(图 3(b)).

最后, 我们通过沿着分子结对称 轴旋转右管 (电极距离固定为 $0.35 \mathrm{~nm}$ ), 研究了两 SWCNT 电极相对取向对 NDR 行为的影响 ${ }^{[15]}$. 很明显, 这样破 坏了分子结的高对称性. 不同旋转角 度的 $I-V$ 曲线和 PVR 值如图 5 所示. 显 然, NDR 行为严重依赖于旋转角度. 当旋转角度从 $0^{\circ}$ 增加到 $72^{\circ}$ 时, PVR 值 从 2.58 下降到 1.09 . 当旋转角度超过 $72 \circ$ 时, NDR 行为消失. 因此, 体系的 对称性最高时可以产生最好的 NDR 行为.

总之, 我们理论研究了电极距离 和相对取向对硼掺杂带帽 SWCNT 分
子结 NDR 行为的影响. 结果显示, NDR 行为强烈依赖于电极距离和相对 取向. 当电极距离为 $0.35 \mathrm{~nm}$ 且体系的

对称性最高时, 可以产生最好的 NDR 行为. 相信我们的结果将有助于 SWCNTs 在 NDR 器件中的应用.
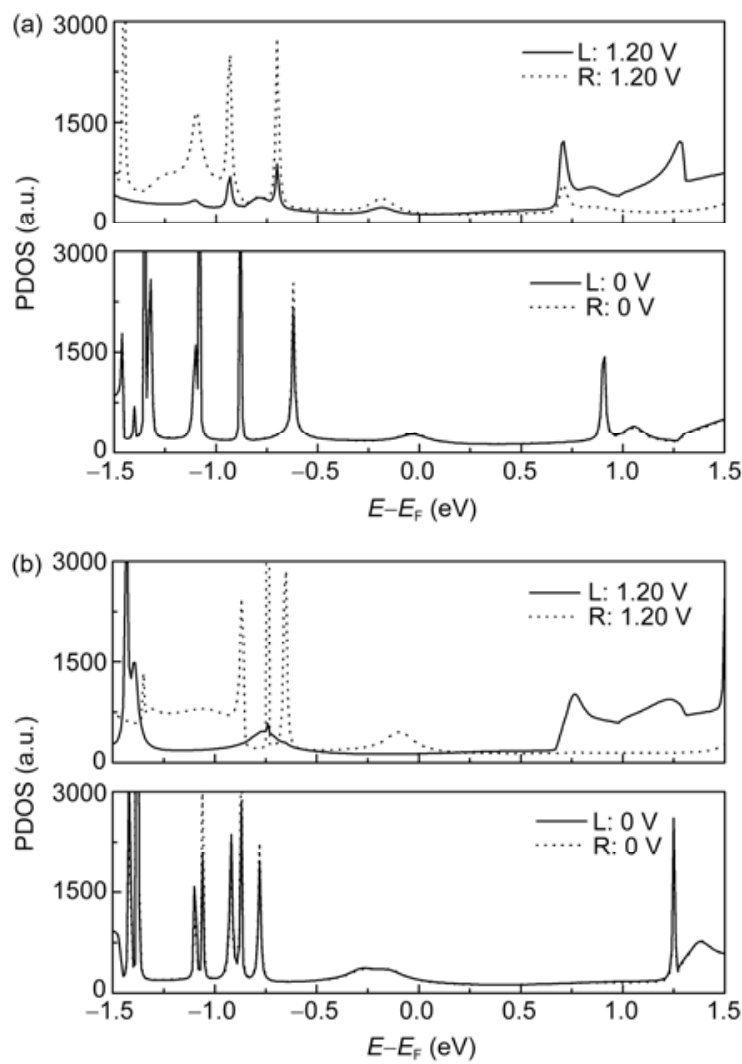

图 4 不同偏压下不同电极距离分子结中左管(实线)和右管(虚线)的 PDOS 谱 (a) $w=0.20 \mathrm{~nm}$; (b) $w=0.35 \mathrm{~nm}$. L 和 $\mathrm{R}$ 分别代表左管和右管

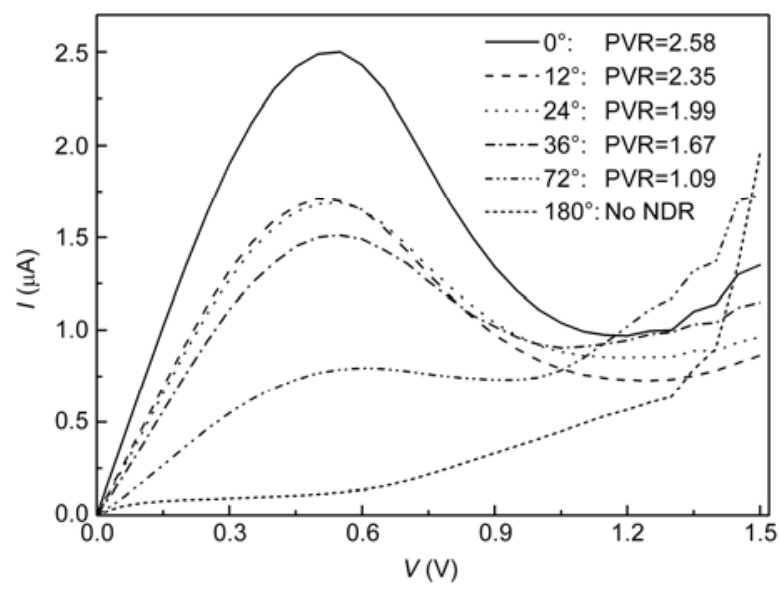

图 56 个不同旋转角度下的 $I-V$ 曲线 两管距离固定为 $0.35 \mathrm{~nm}$ 


\section{参考文献}

1 Andres R P, Bein T, Dorogi M, et al. Science, 1996, 272: 1323-1325

2 Chen J, Reed M A, Rawlett A M, et al. Science, 1999, 286: 1550-1552

3 Long M Q, Chen K Q, Wang L L, et al. Appl Phys Lett, 2008, 92: 243303

4 Zhao P, Fang C F, Xia C J, et al. Appl Phys Lett, 2008, 93: 013113

5 Seminario J M, Araujo R A, Yan L M, et al. J Phys Chem B, 2004, 108: 6915-6918

6 Zhao P, Wang P J, Zhang Z, et al. Phys Lett A, 2010, 374: 1167-1171

7 Zhao P, Liu D S, Zhang Y, et al. Phys Lett A, 2011, 375: 2639-2643

8 Zhao P, Liu D S. Solid State Commun, 2011, 151: 1424-1427

9 Ma J S, Li D M, Zhai Y X, et al. Sci China Phys Mech Astron, 2011, 54: 1433-1437

10 Yang Y T, Song J X, Liu H X, et al. Chin Sci Bull, 2008, 53: 3770-3772

11 Kelly T R, Silva H D. Nature, 1999, 401: 150-152

12 Crljen Ž, Grigoriev A, Wendin G, et al. Phys Rev B, 2005, 71: 165316

13 Ng M K, Lee D C, Yu L P. J Am Chem Soc, 2002, 124: 11862-11863

14 Zhao P, Liu D S. Chin Sci Bull, 2010, 55: 4104-4107

15 Zhao P, Wang P J, Zhang Z, et al. Chin Sci Bull, 2010, 55: 1227-1230

16 Ji G M, Zhai Y X, Fang C F, et al. Phys Lett A, 2011, 375: 1602-1607

17 Wei D C, Liu Y Q, Cao L C, et al. Nano Lett, 2008, 8: 1625-1630

18 Soler J M, Artacho E, Gale J D, et al. J Phys: Condens Matter, 2002, 14: 2745-2779

19 Taylor J, Guo H, Wang J. Phys Rev B, 2001, 63: 245407

20 Brandbyge M, Mozos J L, Ordejón P, et al. Phys Rev B, 2002, 65: 165401

21 Reed M A, Chen J. Chem Phys, 2002, 281: 127-145

22 Büttiker M, Imry Y, Landauer R, et al. Phys Rev B, 1985, 31: 6207-6215 